\title{
An efficient and accurate GB-SAR imaging algorithm based on the fractional Fourier transform
}

\author{
Lilong Zou, Member, IEEE and Motoyuki Sato, Fellow, IEEE
}

\begin{abstract}
In this paper, an efficient and accurate imaging algorithm is presented for Ground-Based Synthetic Aperture Radar (GB-SAR) or other radar systems that could be formed by a physical or synthetic linear aperture. The imaging algorithm is based on the fractional Fourier transform for the azimuth compression. A mathematical framework is derived according to the projection of a sample reflectivity image onto the pseudopolar coordinate and its implementation was presented. With the data acquisition geometry and the pseudopolar imaging coordinate, the phase of a point target can be expressed as a quadratic phase exponential. It makes that only one-dimensional fractional Fourier transform is needed for the azimuth compression of the time domain backscatter data for the GB-SAR imaging problem. By further research, the optimal transformation order which represents the spatial frequency changes by the fractional Fourier transform was given subsequently. Taking advantage of this optimal representation, the proposed approach avoids the large calculation that occurs in the time domain back projection (TDBP). Comparing to the far-field pseudopolar format algorithm (FPFA), the accuracy of the proposed algorithm is much improved. Meanwhile, the proposed approach holds the almost same computational cost and complexity as the FPFA. The proposed approach keeps the advantages of the imaging quality of the TDBP and the computational cost of the FPFA that are two important aspects of the GB-SAR applications. Both the numerical simulation and the field GB-SAR experiment show that the algorithm is more suitable for the high precision GBSAR imaging, especially for the near-field.
\end{abstract}

Index Terms-Ground based synthetic aperture radar (GBSAR), imaging algorithm, the fractional Fourier transform (FrFT), near-filed imaging, linear aperture radar

\section{INTRODUCTION}

G ROUND-based synthetic aperture radar (GB-SAR) is capable of continuous monitoring, providing high sensitivity concerning the terrain deformations. Compared to air- and space-borne SAR, GB-SAR has advantages in the continuous monitoring of targets. Currently, some commercial GB-SAR systems are available. Most of them have a fixed rail of $2 \mathrm{~m}$ length and a radar sensor moving on the linear rail to acquire SAR data with Ku-band $(17 \mathrm{GHz})$ [1][2]. In the last ten years, Tohoku University has applied this technology to the post-landslide monitoring, and a vast number of field campaigns has been carried out in Japan [3][4]. Typically, a site monitoring with GB-SAR instruments produces a large number of data which needs to be focused. For example, the post-landslide monitoring site in Kumamoto with one of our polarimetric GB-SAR system produces a total of $35000 \times 4$ images in an entire year. Moreover, the real-time monitoring is required for the fast movement and the disaster alarm. In one word, an extremely fast and high-resolution imaging algorithm is needed for the environmental monitoring by GBSAR. To cope with the huge volume of data, an accurate focusing algorithm which has more efficient computation is proposed in this paper. This algorithm can also be applied to any radar system which is formed by a physical or synthetic linear aperture.

GB-SAR can be regarded as a linear synthetic aperture radar system. The process of constructing an image from a linear synthetic aperture radar data consists of a two-dimensional compression. First of all, the received echo from each of the acquisition point is compressed. Then the echo is compressed along the azimuth direction by taking advantage of the radar motion in order to synthesize a larger antenna aperture. Nowadays, many imaging algorithms were developed by concerning different purposes and applications. The algorithms consist of two classes: one is the time domain algorithm, the other is the frequency domain algorithm. The time domain algorithm is, as the name implied, focusing in the time domain. The frequency domain algorithm is done in the frequency domain. Both of them have clear advantages and disadvantages.

The time domain methods base on the fact that they can handle an arbitrary system geometry, however, they are slow. The frequency domain methods base on the fact that they are relatively fast by utilizing the fast Fourier transform (FFT), however, they require the sampling positions to lie uniformly on a straight line. Also, the frequency domain methods need a lot of memories to store and evaluate the 2-dimensional Fourier transforms, and the data must be zero-padded to avoid wrap-around effects from the Fourier transforms. There are different algorithms in both of the main groups, all of them are developed to suit for different types of systems, qualities, speed and other criteria.

The frequency domain methods utilizing the FFT are common as the receiver positions which can be modeled to lie on a straight line. The narrowband system and the wideband system must be separated. If working with a narrowband system, we can perform a 2D FFT, a multiplication of the transfer function and a 2D IFFT (the inverse FFT) to construct an image [5]. In this case, a range cell migration compensation has to be applied to account for the non-linear nature of the range samples. This process is called Stolt-interpolation [6][7], too. However, direct implementations of these imaging algorithms are still based on the variant of the range-Doppler (RD) algorithm in the area of SAR processing [8]-[11]. There are also many other popular methods such as the wavenumber algorithm (also called the $\omega-k$ algorithm) [12]-[14] and the chirp scaling algorithm [15]-[17]. All of them perform some kind of range cell migration compensation, but are relatively 
fast due to the use of FFTs.

The time domain methods can also be used in this area. If the image scenes are small or the trajectory of the platform deviates much from a straight, there is no point by the FFTbased method. If there is much motion error, the cost of applying for motion compensation and autofocus algorithms using FFTs is so large that the saving of the computational is lost. The delay-and-sum [18]-[19], back projection (BP) [20]-[25] and Kirchhoff migration (KM) [26] are the relevant algorithms in the time domain.

Back projection (BP) is an exact inversion technique and frequently used for the linear aperture radar imaging. It works in both near and far field, which means that the range of the different contributions is important for finding the focusing delays. To implement this method in practice, the available discrete range samples must be interpolated [11]. Usually, the linear interpolation is used. However, it is possible to apply an advanced interpolator at the expense of the increased computation time. Although this algorithm can handle an arbitrary array geometry and make no approximations, except for the interpolation, it has one major drawback. For each aperture and the pixel position, we need to compute the range between the sensor element and the pixel, interpolate in the received signal and finally add the value found in the image matrix. For a small image, the direct back projection is quite efficient and often preferred due to its simplicity and robustness. However, for the image with a large aperture and size, the expense of the processing time is substantially great.

Another important issue is that whether the GB-SAR is in the near or the far field of the scene. In the near field, the spherical nature of the wave must be taken into account when focusing. If the range satisfies the criteria [27] which is in the far field of the scene, a highly simplified imaging algorithm named far-field pseudopolar format algorithm (FPFA) is proposed in [28]. The FPFA method formats the reflectivity map onto the pseudopolar coordinate and tends to minimize the processing cost. This robust algorithm is frequently used for GB-SAR applications, but it is developed for the far-field imaging.

Therefore, the goal of this paper is to present an efficient and accurate imaging algorithm to suitable the GB-SAR applications. In this paper, a mathematical framework to focus the time domain data by the fractional Fourier transform (FrFT) is developed under the assumption of the linear aperture and the pseudopolar coordinate. Then, the optimal transformation order which represents the spatial frequency changes by the fractional Fourier transform was also given. This optimal representation avoids the large calculation which occurs in the time domain back projection (TDBP). Comparing to the FPFA method, the accuracy of the proposed algorithm is much improved. At the same time, we achieve almost the same computational cost and complexity.

This paper is organized as follows. Section II presents the mathematical framework of the imaging algorithm and the optimization focusing condition. In Section II-A, a brief review, the main applications and the current formulation form of the fractional Fourier transform are introduced. In Section II-B, the mathematical formulation of focusing a GB-SAR image by the FrFT under a pseudopolar coordinate system and the associated coordinate transformations are exhibited. Moreover, the final form of the formulation of the algorithm as an image series is also given in Section II-B. The optimized focusing condition and the optimized rotated angle of the formulation are discussed in Section II-C. Section III presents the comparison of the imaging quality and the computational cost among the TDBP, the FPFA and the proposed approach. The results of an extensive validation of the algorithm with the numerical simulations, the field measurements by GB-SAR data are summarized in Section IV. Finally, the conclusions and the current focus of our research are outlined in Section V.

\section{MATHEMATICAL FRAMEWORK}

\section{A. Fractional Fourier Transform}

In this section, a brief overview of the FrFT and its implementation is presented. The FrFT is first introduced in its current form by Namias [29] in 1979, although the principle underlying FrFT can be found in the work of Wiener and Wely in the 1920s. Later, a rigorous formal study of the FrFT was carried out in [30]. In recent years, the FrFT has received much attention due to its extensive applications in optics [31][32], signal processing [33][34], acoustic wave [35], ultrasound quantum mechanics [36] and pattern recognition [37].

By a complex scaling of a multiplication of a quadratic phase exponential in the transformed domain with the Fourier transform, the exact form of the fractional Fourier transform is shown in Namias [29]. The relation between the angle $\phi$ and the corresponding fractional Fourier transform denoted by

$$
\begin{array}{r}
F_{\phi}(u)=\sqrt{1-j \cot \phi} \cdot \int_{-\infty}^{\infty} e^{j \pi \cot \phi u^{2}} \cdot e^{-j 2 \pi \csc \phi u t} \\
\cdot e^{j \pi \cot \phi t^{2}} \cdot x(t) d t
\end{array}
$$

where $\phi \in\left(-\frac{\pi}{2}, \frac{\pi}{2}\right), t$ is the time and $u$ represent the frequency.

Equation (1) forms the basis for the fractional Fourier transform algorithms. Equation (1) can be simplified as the conventional Fourier transform when $\phi$ is an integer multiple of $\pi / 2$. A physical interpretation of (1) is that it can be realized as a quadratic phase exponential in one domain. The FrFT can transform a signal in the time domain (or in the frequency domain) into the domain between the time and frequency by a rotation.

\section{B. Focusing Formulation}

The specificity of the GB-SAR is the limited length of the synthetic aperture size compared with the conventional SAR. In the typical GB-SAR system such as IBIS-L, FASTGBSAR, the linear rail on the Ku-band with the $300 \mathrm{MHz}$ bandwidth is $2 \mathrm{~m}$, which is equivalent to the fixed $0.5 \mathrm{~m}$ range resolution. The azimuth resolution $\delta_{a}$ depending on the range strongly is defined as:

$$
\delta_{a}=\frac{\lambda}{2 L_{s}} \cdot r
$$


where $\lambda$ is the wavelength, $L_{s}$ is the synthetic aperture length and $r$ is the range distance.

In our model, we set the GB-SAR acquisition geometry as in Fig.1, the platform where the sensor elements are mounted follows a path in the $x$ direction (also called the alongtrack, the azimuth, the cross-range direction, or the slow time domain). The first step is to calculates the reflected signal from a target at the object coordinates $\left(x_{p}, y_{p}\right)$. The position of the transmitting and the receiving antenna on the linear rail is $x_{n}-\frac{d}{2}, x_{n}+\frac{d}{2}$, respectively. We suppose that the spacing $d$ between the receiving and the transmitting element is small enough to be ignored. Here $x_{n}$ is marked as the phase center where the transmitting sensor element sends out a pulse. The time domain reflected signal is compressed as $D\left(x_{n}, t\right)$ in which the received echo from each acquisition point $x_{n}$, where $t$ is the double route delay. Since the electromagnetic waves travel with a much higher speed than that of the platform, $t$ is also called as the fast time domain. Due to the time domain back projection, the synthesis of a radar image can be achieved by integrating the time domain signal with respect to different radar positions $x_{n}$. Therefore, the radar reflectivity map at the point $p$ is estimated as follows:

$$
P\left(x_{p}, y_{p}\right)=\int D\left(x_{n}, t\right) \cdot \exp \left(\frac{4 \pi j R_{n}}{\lambda_{0}}\right) d x_{n}
$$

where $R_{n}$ denotes the double range to the object in meters and $\lambda_{0}$ denotes the wavelength of the system starting frequency.

In this work, we propose an exact mathematical formulation starting from (3) of focusing a GB-SAR image by the FrFT. For the typical GB-SAR system, the amplitude term of the working frequency bandwidth could be ignored, when it varies slowly along the azimuth with certain range. By assuming the pseudopolar coordinate for the object space, the simplest focusing scheme [28] is obtained which is usually adopted for GB-SAR.

The second step is to find an accurate expression for the distance between the target and the sensor

$$
\begin{aligned}
R_{n}\left(x_{n}, x_{p}, y_{p}\right) & =\sqrt{\left(\rho \sin \theta-x_{n}\right)^{2}+(\rho \cos \theta)^{2}} \\
& =\rho \sqrt{1-\frac{2 x_{n} \sin \theta}{\rho}+\frac{x_{n}^{2}}{\rho}} .
\end{aligned}
$$

Applying the first order Taylor expands, (4) can be rewritten as follows:

$$
R_{n}\left(x_{n}, x_{p}, y_{p}\right) \simeq\left(\rho-x_{n} \sin \theta+\frac{x_{n}^{2}}{2 \rho}\right)
$$

by the approximation $\left|\left(2 x_{n} \sin \theta-x_{n}^{2}\right) / \rho\right|<1$. Therefore, the focusing formation could be rewritten as:

$$
\begin{array}{r}
P(\rho, \theta)=\int D\left(x_{n}, t\right) \cdot \exp \left(\frac { 2 \pi j } { \lambda _ { 0 } \rho } \cdot \left(\left(\rho^{2} \sin ^{2} \theta\right.\right.\right. \\
\left.\left.\left.-2 \rho x_{n} \sin \theta+x_{n}^{2}\right)+\left(\rho^{2} \sin ^{2} \theta+2 \rho^{2} \cos ^{2} \theta\right)\right)\right) d x_{n} \\
P(\rho, \theta)=\exp \left(\frac{2 \pi j \rho}{\lambda_{0}}\left(1+\cos ^{2} \theta\right)\right) \int D\left(x_{n}, t\right) . \\
\exp \left(\frac{2 \pi j}{\lambda_{0} \rho}\left(\rho \sin \theta-x_{n}\right)^{2}\right) d x_{n} .
\end{array}
$$

When considering that $\rho \sin \theta=s_{1} u$ and $x_{n}=s_{2} v$, the focusing formation can be expressed as follows:

$$
\begin{array}{r}
P(\rho, \theta)=s_{2} \cdot e^{j \frac{2 \pi \rho}{\lambda_{0}}\left(1+\cos ^{2} \theta\right)} \cdot \int D(v, t) \\
\cdot e^{j \frac{2 \pi}{\lambda_{0} \rho}\left(s_{1}^{2} u^{2}-2 s_{1} s_{2} u v+s_{2}^{2} v_{2}\right)} d v .
\end{array}
$$

where $s_{1}$ and $s_{2}$ are the real value of the scale parameters.

Finally, we introduce a new parameter $g$ to adjust the exponential term in the above formation. Until now, the focusing formation can be written as follows:

$$
\begin{array}{r}
P(\rho, \theta)=s_{2} \cdot e^{j \frac{2 \pi \rho}{\lambda_{0}}\left(2-g \sin ^{2} \theta\right)} \cdot \int D(v, t) \\
\cdot e^{j \frac{2 \pi}{\lambda_{0} \rho}\left(g s_{1}^{2} u^{2}-2 s_{1} s_{2} u v+s_{2}^{2} v_{2}\right)} d v .
\end{array}
$$

By comparing this formation with the definition of the FrFT (1), we conclude that $P(\rho, \theta)$ is proportional to the FrFT at the position $x_{n}$, i.e.,

$$
P(\rho, \theta)=A \cdot F_{\phi}\left(\frac{x_{n}}{s_{2}}\right)
$$

with

$$
A=\frac{s_{2} \cdot \exp \left(\frac{2 \pi j \rho}{\lambda_{0}}\left(2-g \sin ^{2} \theta\right)\right)}{\sqrt{1-j \cot \phi}} .
$$

Here $A$ is a constant for the certain range. Equation (10) holds if and only if

$$
\begin{aligned}
& \cot \phi=\frac{2 g \cdot s_{1}^{2}}{\lambda_{0} \cdot \rho}, \\
& \csc \phi=\frac{2 s_{1} \cdot s_{2}}{\lambda_{0} \cdot \rho}, \\
& \cot \phi=\frac{2 s_{2}^{2}}{\lambda_{0} \cdot \rho} .
\end{aligned}
$$

Since $\cot ^{2} \phi+1=\csc ^{2} \phi$, we obtain

$$
s_{1}^{4}=\frac{\lambda_{0}^{2} \cdot \rho^{2}}{4 g(1-g)}
$$

which yields

$$
0<g<1
$$

For a given range $\rho$ and the rotated angle $\phi$ of the fractional Fourier transform, we notice that the parameters $g,\left|s_{1}\right|$ and $\left|s_{2}\right|$ are determined uniquely. Equation (12) yields that $\cot \phi>$ 0 . Therefore, we have

$$
0<\phi<\frac{\pi}{2}
$$

So far, we have obtained a new focusing formation (9) and the corresponding necessary conditions $0<g<1$ and $0<$ $\phi<\pi / 2$. 


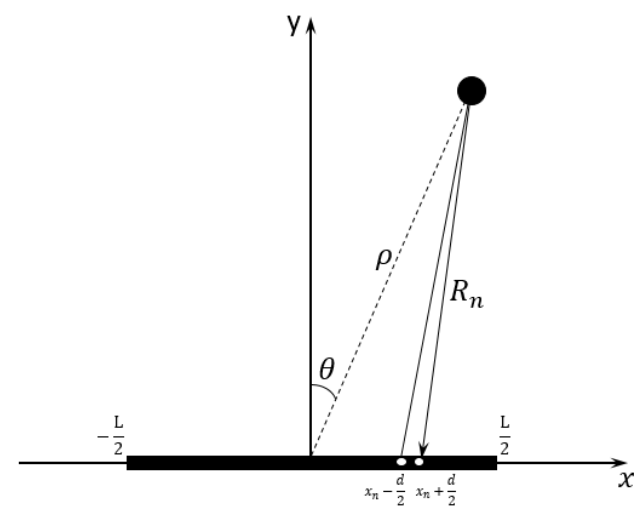

Fig. 1. GB-SAR acquisition geometry.

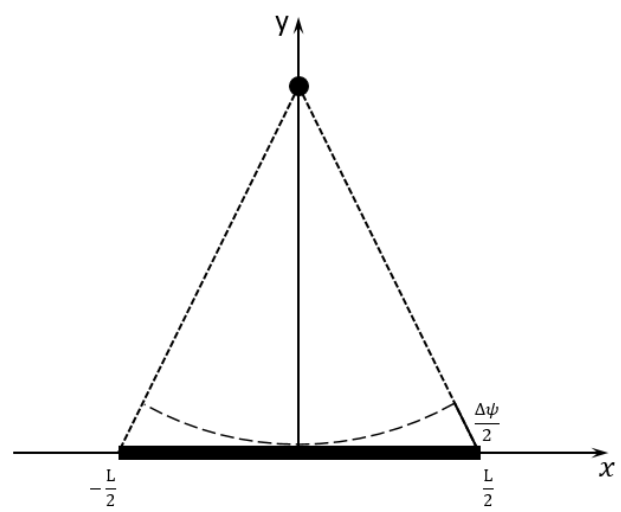

Fig. 2. Relationship between the phase of one single target and the position on the linear rail at a certain range.

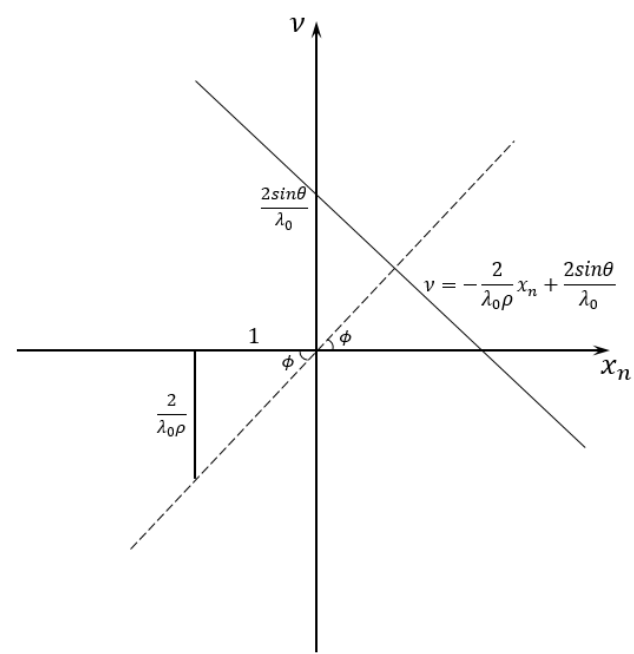

Fig. 3. Geometric interpretation of the relationship between the optimal rotated angle of fractional Fourier transform and the instantaneous spatial frequency.

\section{Optimal Focusing Condition}

In Section B, we present the proposed mathematical formation and the necessary conditions for focusing a GB-SAR image. In this section, we focus on the optimization focusing condition. Furthermore, we compare our computational cost to that of the FPFA.

There is a point target in the line of the sight (LOS) direction

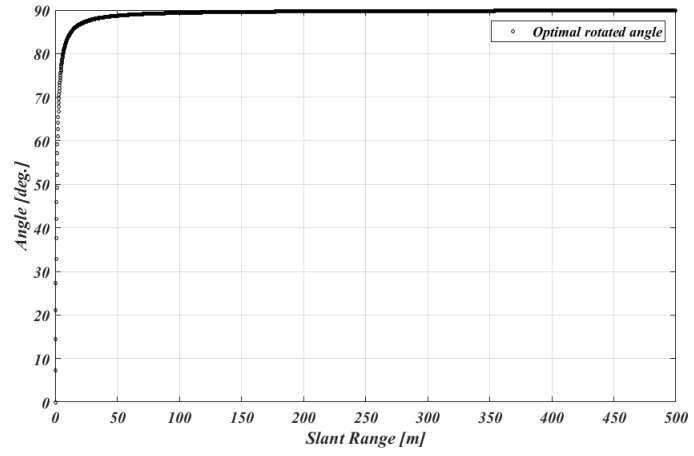

Fig. 4. The optimal rotated angle of the FrFT for a certain range by considering the IBIS-L GB-SAR system.

shown in Fig. 2. At each position along the rail, the point targets contribute to the same amplitude for all the reflections in the range resolution. However, the phase $\psi$ is different slightly for the distance from the antenna to the target. In the case of GB-SAR, the phase $\psi$ at the position $x_{n}$ of the antenna can be expressed as follows:

$$
\psi\left(x_{n}\right)=-\frac{4 \pi}{\lambda_{0}} \cdot \sqrt{x_{n}^{2}+y_{p}^{2}} .
$$

The spatial frequency $\nu$ are the spatial analog of the angular velocity $\omega$ and the frequency $f$ in the time domain of a signal. In this paper, we rewrite the spatial frequency $\nu$ with regard to the phase $\psi$ in the polar coordinate as follows:

$$
\nu\left(x_{n}\right)=\frac{k\left(x_{n}\right)}{2 \pi}=\frac{1}{2 \pi} \cdot \frac{d \psi\left(x_{n}\right)}{d x_{n}} \simeq \frac{2 \sin \theta}{\lambda_{0}}-\frac{2 x_{n}}{\lambda_{0} \cdot \rho},
$$

where $k$ is the angular wavenumber. Hence the spatial frequency $\nu$ is linear to the antenna position for a certain range.

As the range increasing, the difference of the instantaneous spatial frequency at different antenna position decrease. And the variety of this spatial frequency will produce a greater blur in the traditional Fourier based focusing algorithm. However, the distribution of the above linear spatial frequency has the narrowest representation in the fractional domain. Thus, by finding the optimal rotated angle $\phi$ of the FrFT we can provide a highly focused response in the azimuth direction of a certain range signal.

The solid line in Fig.3 indicates the variety of the spatial frequency at different antenna position $x_{n}$. As the axis (the dashed line) rotates to a position perpendicular to the solid line, then the magnitude response reaches the maximum value. For the simplest case, the optimal angle is defined as follows:

$$
\phi_{o p t}=\tan ^{-1}\left(\frac{\lambda_{0} \cdot \rho}{2}\right) .
$$

From an observation of the real GB-SAR, the data is discrete along the linear rail. Using the interpretation shown in Fig.3 we obtain

$$
\phi_{o p t}=\tan ^{-1}\left(\frac{\Delta \nu \cdot \lambda_{0} \cdot \rho}{2 \Delta x_{n}}\right),
$$

where $\Delta \nu$ is the spatial sampling frequency; $x_{n}$ is the step size of the scan along the linear rail and $N$ denote the number 
of the acquisition points along the linear rail. Since $\Delta \nu=$ $\Delta x_{n} / N$, we have

$$
\phi_{o p t}=\tan ^{-1}\left(\frac{\lambda_{0} \cdot \rho}{2 N \cdot \Delta x_{n}^{2}}\right) .
$$

Equation (22) shows the optimal rotated angle of the FrFT for a certain range of the GB-SAR data, which means that we can get an an optimal rotated angle by (22) for any range of the data in the GB-SAR observation. The optimal rotated angle is determined by the starting frequency, the size of the step and the number of the acquisition of the GB-SAR system setting. Moreover, it is also determined by the slant range. Using the optimal rotated angles with (11)-(13), the parameters $s_{1}, s_{2}$ and $g$ which are used to focus a GB-SAR image by the proposed FrFT approach can be calculated.

The distance of the range from the radar to any arbitrary points within the image scene is denoted as $\rho^{\prime}$. Then the far field criteria of the radar aperture is

$$
\rho^{\prime}>\frac{2 L^{2}}{\lambda_{c}},
$$

where $\lambda_{c}$ is the wavelength at the center frequency of the radar. For a typical commercial GB-SAR system working at Ku-band with $5 \mathrm{~mm}$ step size along the $2 \mathrm{~m}$ linear rail, the far field criteria is around $460 \mathrm{~m}$. The optimal rotated angle calculated by (22) is presented in Fig.4. The rotated angle changes from 0 to 90 degree until the range distance reach the criteria. When the range distance satisfies the far field criteria, the proposed approach match to the FPFA.

\section{IMAGE QUALITY AND COMPUTATIONAL COST}

This section presents the performance of our proposed approach. The quantitative analysis is carried out from the quality of the images and the speed of the algorithm. Moreover, we compare our algorithm with the TDBP and the FPFA.

\section{A. Image Quality}

It is important to establish the GB-SAR image quality in different imaging algorithms. There are several measures used to judge the quality of an image. In this paper, we focus on the point spread functions in the azimuth direction, and calculate the peak to the sidelobe ratio (PSLR) and the integrated sidelobe ratio (ISLR). PSLR is the ratio between the peak of the main lobe and that of the most prominent side lobes [38]. ISLR is defined as the ratio between the energy of the main lobe and that integrated over all the side lobes [39]. Both sides of the main lobe in azimuth direction in a range resolution, are calculated in this paper. Since the extent of the scene is limited, we typically integrate over several (5 to 10) lobes on both sides of the main one.

In the following simulations, we compare our approach to two different algorithm on the imaging of one reflector: the TDBP and the FPFA. The position of the reflector changes from $3 \mathrm{~m}$ to $500 \mathrm{~m}$ in range. The parameters of a real GBSAR system are used in the simulations. Figure 5 and 6 show PSLR and ISLR of the reflector for the TDPB, the FPFA and the proposed approach, respectively. For the proposed

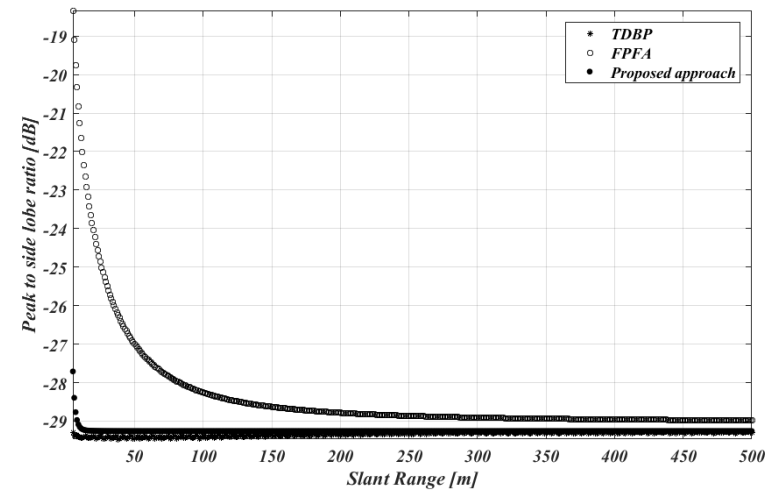

Fig. 5. Peak to side lobe ratio of the TDBP, the FPFA and the proposed approach.

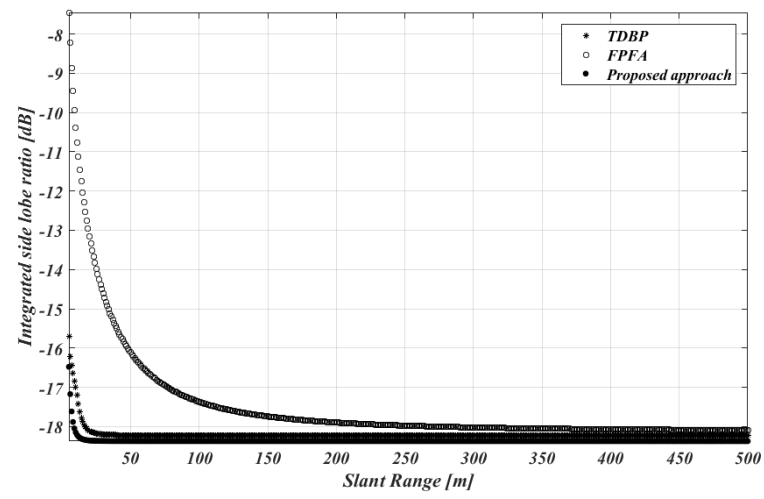

Fig. 6. Integrated side lobe ratio of the TDBP, the FPFA and the proposed approach.

approach, the criteria $\left|\left(2 x_{n} \sin \theta-x_{n}^{2}\right) / \rho\right|$ is approximately $10 \mathrm{~m}$ in range. When the slant range satisfies the criteria, $\operatorname{PSLR}(\approx-29 d B)$ and $\operatorname{ISLR}(\approx-18 d B)$ do not change much between the proposed approach and the TDBP. Notably, PSLR and ISLR of the FPFA move close to that of the TDBP with the range increasing until to the far field condition. Therefore, when the target position satisfies the approximated criteria, the image quality by the proposed approach shows the main lobe equally comparing to that of the TDBP. It is worth mentioning that the criteria satisfies most of the application conditions. Now, the typical criteria given for the current commercial GBSAR system is around $10 \mathrm{~m}$.

\section{B. Computational Cost}

The synthesis of an entire reflectivity image using (3) has associate a high computational cost defined by $O\left(N N^{\prime} M^{\prime}\right)$, where $M^{\prime}$ and $N^{\prime}$ denote the number of pixels in the $x$ and $y$ directions, respectively. $N$ denotes the acquisition point along the linear rail. In practice, the TDBP need the interpolation before the azimuth compression. Typically, an FFT with zero padding will be used.

The proposed approach based on the FrFT has the similar properties as the FPFA. Before focusing the GB-SAR image by (9), the 1-D Fourier transform is needed to transform the 


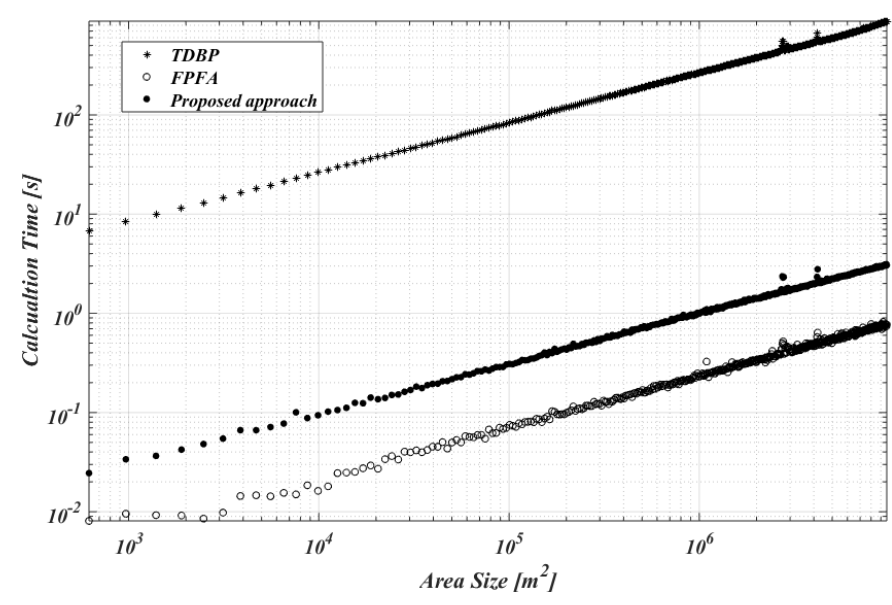

Fig. 7. Computation time of the TDBP, the FPFA and the proposed approach.

signals from the frequency domain to the time domain. Then 1D fractional Fourier transform need to be used for the azimuth compression. Ozaktas (1996) [40] presented an efficient and accurate computation algorithm of the FrFT which has the same computational cost as the FFT. In the proposed approach, the total computational cost from the raw data to the image is $O(M N \log M N+M(N+4))$. Here $M$ is the number of the frequency points and $N$ is the number of the acquisition points along the linear rail. $O(M N \log M N)$ is the sum of the computational cost in 1-D FFT for the range compression and 1-D fractional Fourier transform for the azimuth compression. And $O(M(N+4))$ is the computational cost for the calculation of the parameters. When the parameters are given fixed, we use them repeatedly for a GB-SAR measurement with the fixed acquisition parameters. For example, if we focus a real GB-SAR measurement with the scene size of $500 \mathrm{~m}$ in the range and $400 \mathrm{~m}$ in the azimuth, the computational cost of the proposed approach is 50 times lower than that of the TDBP. However, for the FPFA, no parameters need to be calculated beforehand and the computational cost is $O(M N \log M N)$.

To test the computational cost of the algorithms, we use an Inter(R) Core(TM) i7-6700 CPU@3.40GHz with 32GB RAM Desktop PC with MATLAB. The results of the simulations are shown in Fig. 7, which show the comparison for different size of the scene focusing by the TDBP, the FPFA and the proposed FrFT algorithm presented in Section B.The proposed FrFT approach save much more time than the TDBP which can be observed in Fig. 7. However, the proposed FrFT approach costs more computational time than the FPFA. That is due to two factors: first, the parameters in the proposed FrFT approach do not appear in the FPFA. Second, the Fourier transform function in the FPFA is more efficient in MATLAB. Up to now, we have proved that the proposed FrFT approach can improve the computational cost comparing to the TDBP.

\section{Results}

The previous section analyzed the performance and the accuracy of our proposed FrFT algorithm. In this section, the efficiency of our algorithm is analyzed using both the numerical simulation and the field experiment. Moreover, we compare

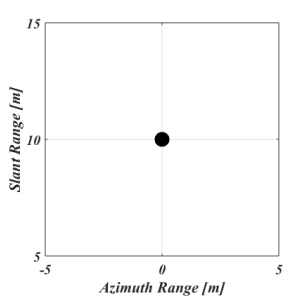

(a)

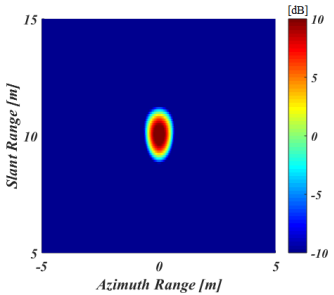

(c)

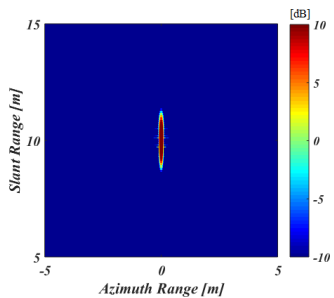

(b)

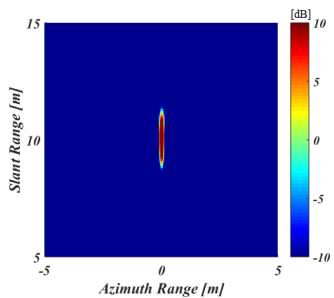

(d)
Fig. 8. Simulation model and focused GB-SAR image by different methods; (a) a point target located at $10 \mathrm{~m}$ in range; (b) the TDBP; (c) the FPFA; (d) the proposed approach.

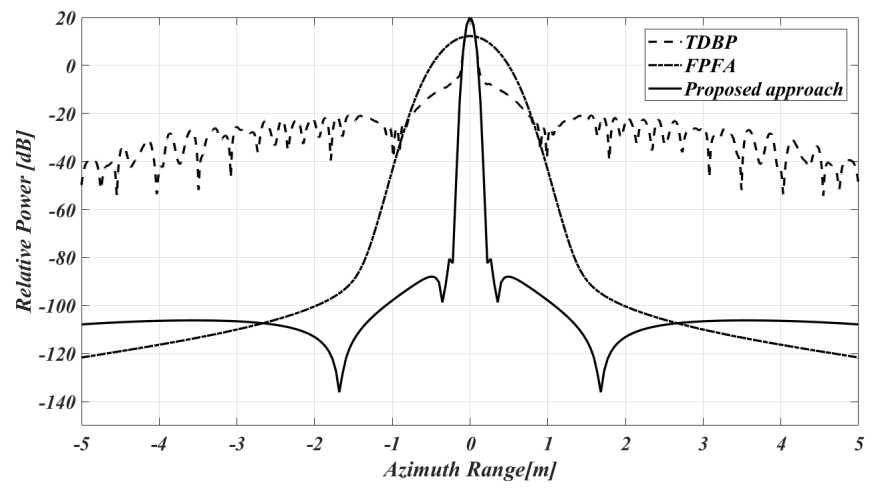

Fig. 9. Azimuth cuts of reflectivity image at range $10 \mathrm{~m}$ obtained by the TDBP, the FPFA and the proposed approach, respectively.

the quality of the focused images obtained by the proposed FrFT approach, the TDBP, and the FPFA, respectively.

\section{A. Numerical Simulation}

The parameters of the simulation are given the same as the real GB-SAR system. The radar system operates at 17 $\mathrm{GHz}$ (Ku-band) carry frequency and $300 \mathrm{MHz}$ bandwidth and the aperture size is $2 \mathrm{~m}$. Before focusing the image, Hanning window function has been applied to the data both in the frequency domain and the time domain. The reflectivity images using the TDBP, the FPFA, and the proposed FrFT approach are obtained, respectively.

Define a scene consisting of a point scatter at $10 \mathrm{~m}$ in the range direction and $0 \mathrm{~m}$ in the azimuth direction as shown in Fig.8(a). The focused image by the TDBP, the FPFA and the proposed approach are shown in Fig.8 (b)-(d), respectively. The focused image by the FPFA and the proposed approached are plotted in the pseudopolar coordinate, while the focused image by the TDBP is plotted in the Cartesian coordinate. As it can be seen from Fig. 8, the point scatter by the proposed 


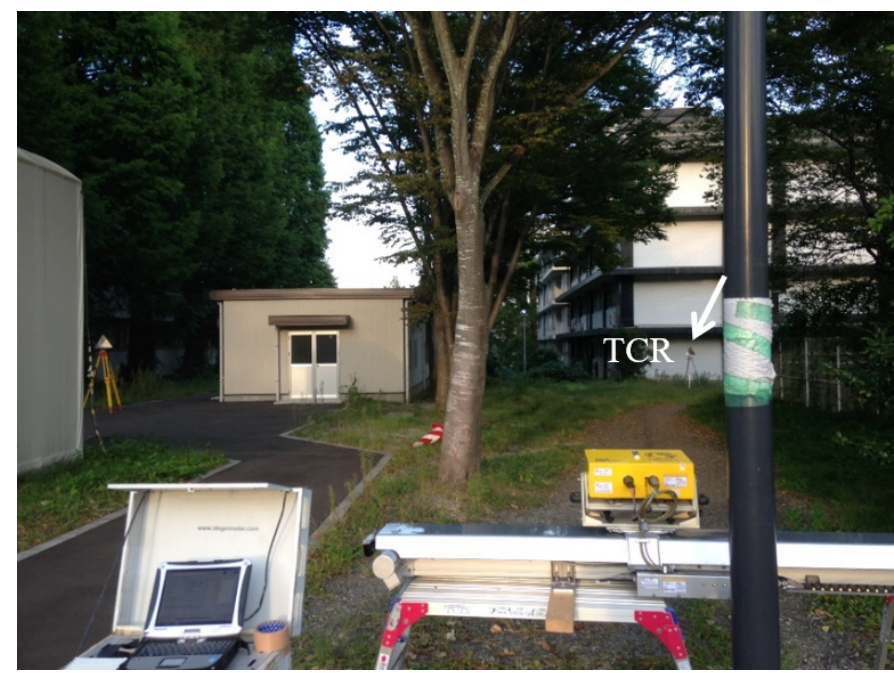

Fig. 10. GB-SAR observation at Kawauchi Campus, Tohoku University. The red vector indicate the trihedral corner reflector.

TABLE I

SPECIFICATIONS OF IBIS-L GB-SAR SYSTEM

\begin{tabular}{cc}
\hline Parameter & Value \\
\hline Central Frequency $f$ & $17.175 \mathrm{GHz}$ \\
\hline Central Wavelength $\lambda$ & $17.44 \mathrm{~mm}$ \\
\hline Bandwidth $B$ & $300 \mathrm{MHz}$ \\
\hline Scan Length $L_{s}$ & $2 \mathrm{~m}$ \\
\hline Scan time $\Delta t$ & $5 \mathrm{~min}$ \\
\hline Maximum Distance $R_{\max }$ & $4000 \mathrm{~m}$ \\
\hline Range Resolution $\delta_{r}$ & $0.5 \mathrm{~m}$ \\
\hline Cross-Range Resolution $\delta_{c}$ & $4.4 \mathrm{mrad}(0.44 \mathrm{~m} \mathrm{~m}$ at $100 \mathrm{~m}$ range $)$ \\
\hline
\end{tabular}

approach is well focused. We investigate the result by cutting the azimuth of the reflectivity image at $10 \mathrm{~m}$ range as shown in Fig. 9. It can be observed that the azimuth resolution of the point scatter focused by the proposed approach shows small differences to that by the TDBP. Therefore, the results clearly show that the proposed approach is an excellent method.

\section{B. GB-SAR Measurement}

Also, the algorithm is tested on the data of the real experiment acquired by IBIS-L GB-SAR system. The site is located at the Kawauchi campus of Tohoku University, Sendai, Japan, as shown in Fig.10. The IBIS-L GB-SAR system used in this study features two horn antennas, one for transmitting and the other for receiving, both with vertical polarization. The system operates in the Ku-band with the center frequency of $17.175 \mathrm{GHz}$ and the bandwidth of $300 \mathrm{MHz}$. The radar is a stepped-frequency system with variable frequency sampling points that are determined on the basis of the observational range. The entire radar-and-antenna assembly is mounted on a linear rail and it scans about $2 \mathrm{~m}$ repeatedly. The $2 \mathrm{~m}$ scan spends two minutes and it is repeated every 5 minutes. The system acquires data every $5 \mathrm{~mm}$ along a $2 \mathrm{~m}$ scan length at 401 azimuth positions. The rest parameters of the system are summarized in Table I.

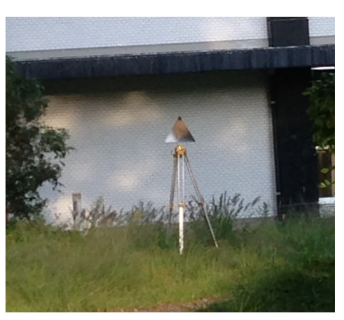

(a)

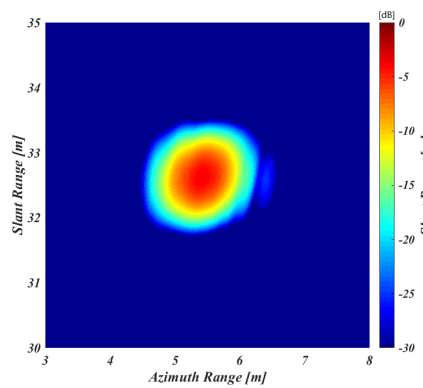

(c)

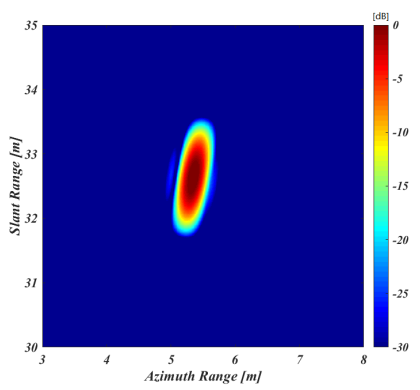

(b)

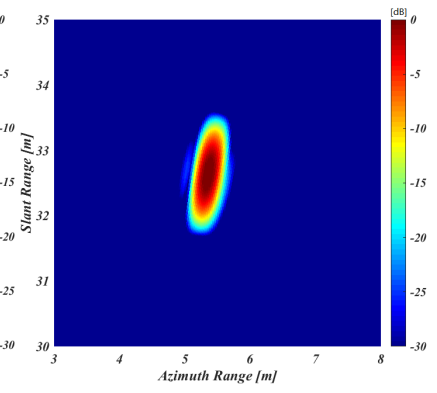

(d)
Fig. 12. Trihedral Corner Reflector and focused image by different methods; (a) a trihedral corner reflector; (b) the TDBP; (c) the FPFA; (d) the proposed approach.

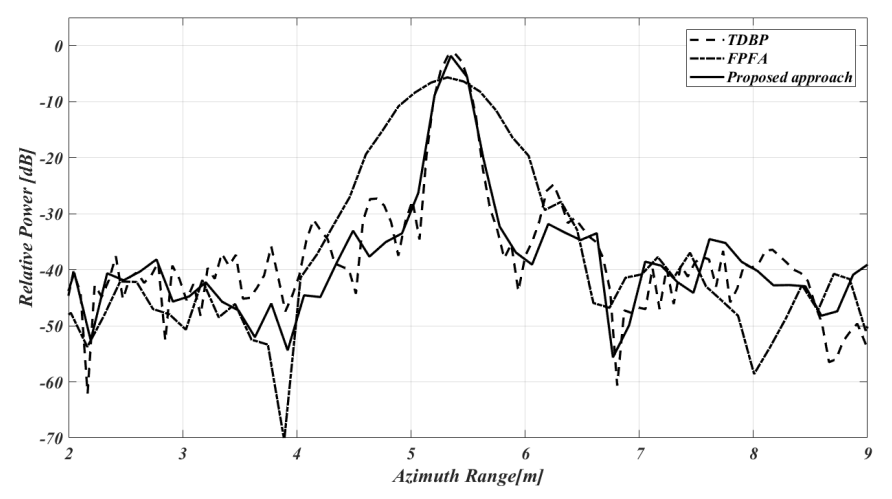

Fig. 13. Azimuth cuts of reflectivity image at range $30 \mathrm{~m}$ obtained by the TDBP, the FPFA and the proposed approach, respectively.

A trihedral corner reflector (TCR) is placed about $33 \mathrm{~m}$ away from the center of the linear rail, shown in Fig.10 by the white arrow. This reflector is mounted on a tripod and the height is adjusted to be on the radar line of sight as shown in Fig.12(a). The focused GB-SAR images obtained by the TDBP, the FPFA and the proposed approach are shown in Fig.11 (a)-(c), respectively. The white circles in Fig.11 indicate the TCR discussed above. The enlarged image that of the TCR by the TDBP, the FPFA and the proposed approach are shown in Fig.12 (b)-(d), respectively. The azimuth cuts of the TCR located at 33 meters in range is shown in Fig. 13. From these results, it is obvious that the image focused by the proposed approach shows equally main lobe compare to that by the TDBP, while the FPFA produces defocusing in both the azimuth and the range direction since the far 


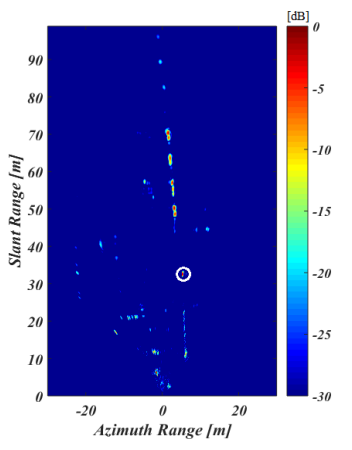

(a)

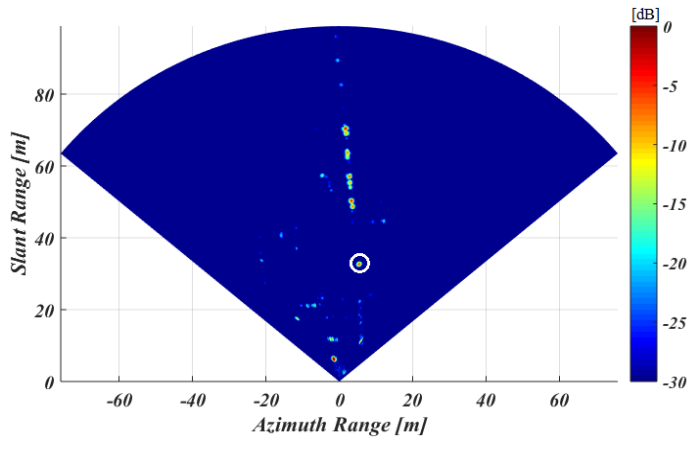

(b)

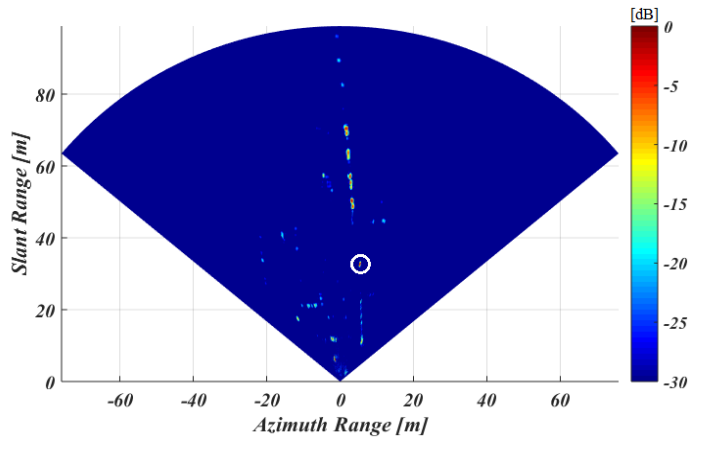

(c)

Fig. 11. Focused GB-SAR image by different methods; (a) the TDBP; (b) the FPFA; (c) the proposed approach.

range approximations do not hold for the target position here considered.

\section{CONClusion}

In this paper, we report an efficient and accurate imaging algorithm for GB-SAR. The algorithm is suitable for any radar systems that could form as a physical or synthetic linear aperture. This technique is a type of modified time domain back projection and focuses on the reflectivity map of a polar coordinate. By purely considering the geometrical coordinate and the spatial phase of a fixed target in the linear antenna aperture, it is clearly shown that the azimuth phase mechanism could be realized as a quadratic phase exponential. The quadratic phase exponential satisfies the form of the fractional Fourier transforms so that only the 1-D fractional Fourier transform is required to the azimuth compression. Relies on the geometry of the imaging scenario, the spatial frequency along the line rail which is related to the quadratic phase exponential can be optimally represented by the fractional Fourier transform with a certain rotated angle. Considering the real GB-SAR system acquisition parameters, the optimal focusing condition is also given in this paper. Moreover, we compare our algorithm from the imaging quality and the computational cost. Compared with the TDBP, the proposed approach obtained almost the same imaging quality, while the computational cost is extremely low. Compared to the FPFA, computational cost of the proposed approach is almost identical, while the proposed approach also provide accurate imaging results, especially in the near-field. The numerical simulations and the field GB-SAR experiments show that the accurate image can be obtained by the proposed approach no matter in the near- or the far-field. Furthermore, our approach has lower computational cost, which is an important requirement for the GB-SAR applications.

\section{REFERENCES}

[1] O. Monserrat, M. Crosetto, and G. Luzi, "A review of ground-based sar interferometry for deformation measurement," ISPRS J. Photogramm. Remote Sens., vol. 93, pp. 40-48, Jul. 2014.

[2] S. Rodelsperger, A. Coccia, D. Vicente, C. Trampuz, and A. Meta, "The Novel fastGBSARSensor: Deformation Monitoring for Dike Failure Prediction," in APSAR 2013, Tsukuba,Japan, Sep. 2013, pp. 23-27.
[3] L. Zou, K. Takahashi, and M. Sato, "Displacement measurement and monitoring with GB-SAR; case study at Aratozawa," in Proc. of Microwave Conference(APMC), 2014 Asia-Pacific, Sendai, Japan, Nov. 2014, pp. 1022-1024.

[4] K. Takahashi, M. Matsumoto, and M. Sato, "Continuous Observation of Nature-Disaster-Affected areas using Ground-Based SAR Interferometry," IEEE J. Sel. Topics Appl. Earth Observ. Remote Sens., vol. 6, no. 3, pp. 1286-1294, 2013.

[5] D. C. Munson and R. L. Visentin, "A Signal processing view of stripmapping synthetic aperture radar," IEEE Trans. Acoust., Speech, Signal Process., vol. 37, no. 12, pp. 2131-2147, Dec. 1989.

[6] C. Cafforio, C. Prati, and F. Rocca, "SAR data focusing using seismic migration techniques," IEEE Trans. Aerosp. Electron. Syst., vol. 27, pp. 194-207, Feb. 1991.

[7] A. Li, "Algorithms for the implementation of Stolt interpolation in SAR processing," in Proc. IEEE IGARSS, Houston, Unite State, May 1992, pp. 360-362.

[8] I. G. Cumming and F. H. Wong, Digital Processing of Synthetic Aperture Radar Data: Algorithms and Implementation. MA: Norwood: Artech House, 2005.

[9] R. Bamler, "A comparison of range-doppler and wavenumber domain SAR focusing algorithms," IEEE Trans. Geosci. Remote Sensing, vol. 30, pp. 706-713, July 1992.

[10] A. M. Smith, "A new approach to range-Doppler SAR processing," Int. J. Remote Sensing, vol. 12, pp. 235-251, 1991.

[11] M. Y. Jin and C. Wu, "A SAR correlation algorithm which accommodates large range migration," IEEE Trans. Geosci. Remote Sensing, vol. GE-22, pp. 592-597, 1984.

[12] R. W. et al., "Focus FMCW SAR data using the wavenumber domain algorithm," IEEE Trans. Geosci. Remote Sensing, vol. 48, no. 4, pp. 2109-2118, April 2010.

[13] I. G. Cumming, Y. L. Neo, and F. H. Wong, "Interpretations of the OmegaK algorithm and comparisons with other algorithms," in Proc. IEEE IGARSS, Toulouse, French, July 2003, pp. 1455-1458.

[14] A. M. Guarnieri and S. Scirpoli, "Efficient Wavenumber Domain Focusing for Ground-Based SAR," IEEE Geosci. Remote Sens. Lett., vol. 7, no. 1, pp. 161-165, 2010.

[15] R. K. Raney, H. Runge, R. Bamler, I. Cumming, and F. Wong, "Efficient Wavenumber Domain Focusing for Ground-Based SAR," IEEE Geosci. Remote Sens. Lett., vol. 7, no. 1, pp. 161-165, 2010.

[16] R. S. A. Moreira, J. Mittermayer, "Extended chirp scaling algorithm for air- and spaceborne SAR data processing in stripmap and ScanSAR imaging modes," IEEE Trans. Geosci. Remote Sensing, vol. 34, pp. 1123-1136, Sept. 1996.

[17] A. Moreira and Y. Huang, "Airborne SAR processing of highly squinted data using a chirp scaling approach with integrated motion compensation," IEEE Trans. Geosci. Remote Sensing, vol. 32, pp. 1029-1040, Sept. 1994.

[18] J. McCorkle and M. Rofheart, "An order N2log(N) backprojector algorithm for focusing wide-angle wide-bandwidth arbitrary-motion synthetic aperture radar," in SPIE AeroSense Conference, April 1996, pp. 8-9.

[19] L. J. Busse, "Three-dimensional imaging using a frequency-domain synthetic aperture focusing technique," IEEE Trans. Ultrason. Ferroelectr. Freq. Control, vol. 39, no. 2, pp. 174-179, 1993. 
[20] A. F. Yegulalp, "Fast backprojection algorithm for synthetic aperture radar," in Proc. IEEE Radar Conf., April 1999, pp. 60-64.

[21] K. Knaell and G. Cardillo, "Radar tomography for the generation of three-dimensional images," Proc. Inst. Elect. Eng.Radar Sonar Navig., vol. 142, no. 2, pp. 54-60, Apr. 1995.

[22] S. Nilsson and L. E. Andersson, "Application of fast back-projection techniques for some inverse problems of synthetic aperture radar," Proc. SPIE Algorithms Synthetic Aperture Radar Imagery V, vol. 3370, pp. 62-72, Apr. 1998.

[23] L. M. H. Ulander, H. Hellsten, and G. Stenstrom, "Synthetic-aperture radar processing using fast factorized back-projection," IEEE Trans. Aerosp. Electron. Syst., vol. 39, no. 3, pp. 760-776, Jul. 2003.

[24] L. Zhou, C. Huang, and Y. Su, "A fast back-projection algorithm based on cross correlation for gpr imaging," IEEE Geosci. Remote Sens. Lett., vol. 9, no. 2, pp. 228-232, Mar. 2012.

[25] C. Cafforio, C. Prati, and F. Rocca, "SAR data focusing using seismic migration techniques," IEEE Trans. Aerosp. Electron. Syst., vol. 27, no. 2, pp. 194-207, Mar. 1991.

[26] S. Basu and Y. Bresler, "Error analysis and performance optimization of fast hierarchical backprojection algorithms," IEEE Trans. Image Process., vol. 10, no. 7, pp. 1103-1117, Jul. 2001.

[27] F. M. Henderson and A. J. Lewis, Principles and Applications of Imaging Radar. New York: Wiley, 1998, vol. 2.

[28] J. FortunyGuasch, "A fast and accurate far-field psedopolar format radar imaging algorithm," IEEE Trans. Geosci. Remote Sens., vol. 47, no. 4, pp. 1187-1196, 2009.

[29] V. Namias, "The fractional order Fourier transform and its application to quantum mechanics," J. Ins. Math. Appl., vol. 25, no. 3, pp. 241-265, 1980.

[30] A. C. McBride and F. H. Kerr, "On Namias's fractional Fourier transform," IMA J. Appl. Math, vol. 39, no. 2, pp. 159-175, 1987.

[31] A. W. Lohmann, "Image rotation Wigner rotation and the fractional order Fourier transform," J. Opt. Soc. Amer. A., vol. 10, pp. 2181-2186, 1993.

[32] D. Mendlovic and H. M. Ozaktas, "Fractional Fourier transforms and their optical implementation 1," J. Opt. Soc. Amer. A., vol. 10, pp. 1875$1881,1993$.

[33] L. B. Almeida, "The fractional Fourier transform and time-frequency representations," IEEE Trans. Signal Processing, vol. 42, pp. 3084-3091, Nov. 1994.

[34] H. M. O. M. F. Erden, M. A. Kutay, "Repeated filtering in consecutive fractional fourier domains and its application to signal restoration," IEEE Trans. Signal Processing, vol. 47, pp. 1458-1462, May 1999.

[35] G. Gonon, O. Richoux, and C. Depollier, "Acoustic Wave Propagation in a 1-D Lattice: Analysis of Nonlinear Effects by the Fractional Fourier Transform Method," Signal Process., vol. 83, pp. 2469-2480, 2003.

[36] M. Arif, D. M. J. Cowell, and S. Freear, "Pulse compression of harmonic chirp signals using the fractional fourier transform," Ultrasound Med. Biol., vol. 36, no. 6, pp. 949-956, 2010.

[37] W. Jin and Y. Zhang, "Color pattern recognition based on the joint fractional Fourier transform correlator," Chinese Optics Lett., vol. 5, no. 11 , pp. $628-631,2007$.

[38] D. Massonnet and J. C. Souyris, Imaging with Synthetic Aperture Radar. Boca Raton: FL: CRC, 2008.

[39] M. A. and J. Marchand, "Sar image quality assessment," Asoc. Espaola de Teledeteccin Revista de Teledeteccin, vol. 2, pp. 12-18, 1993.

[40] H. M. Ozaktas, O. Arikan, M. A. Kutay, and G. Boadagi, "Digital computation of the fractional Fourier transform," IEEE Trans. Signal Processing, vol. 44, no. 9, pp. 2141-2150, Sep. 1996. 